\title{
Neuroendocrine differentiation is predictive of poor survival in patients with stage II colorectal cancer
}

\author{
YUE LIU $^{1 *}$, JINJIE HE $^{1 *}$, JINGHONG XU², JUN LI $^{3}$, YURONG JIAO $^{3}$, DIKAI BEI ${ }^{1}$, \\ YETING HU $^{1}$, HAIYAN CHEN ${ }^{1}$, QIAN XIAO ${ }^{1}$ and KEFENG DING ${ }^{3}$ \\ ${ }^{1}$ Cancer Institute; Departments of ${ }^{2}$ Pathology and ${ }^{3}$ Surgical Oncology, Key Laboratory of Cancer Prevention \\ and Intervention, China National Ministry of Education, Key Laboratory of Molecular Biology in Medical Sciences, \\ The Second Affiliated Hospital, Zhejiang University, School of Medicine, Hangzhou, Zhejiang 310009, P.R. China
}

Received January 1, 2016; Accepted November 25, 2016

DOI: $10.3892 / \mathrm{ol} .2017 .5681$

\begin{abstract}
The diagnosis of neuroendocrine differentiation (NED) is made primarily on the basis of ultrastructure and/or immunohistochemistry (IHC). Synaptophysin (Syn) and chromogranin A (CgA) are two important frequently used NED markers in colorectal cancer (CRC). The association between $\mathrm{NED}$ and the prognosis of stage II CRC remains controversial. Administration of adjuvant chemotherapy remains challenging for stage II CRC. Identification of reliable factors that improve the selection of patients with stage II CRC at high risk following surgery is of great importance. A total of 151 cases of patients with stage II CRC who received radical surgery in The Second Affiliated Hospital of Zhejiang University (Hangzhou, China) between January 2002 and March 2011 were assayed for Syn and CgA using IHC, following which patients were classified as $\mathrm{NED}(+)$ or NED(-). Survival curves were constructed using the Kaplan-Meier estimator, and the prognostic value was determined using a log-rank test and Cox's regression test. In the 151 cases of stage II CRC examined, the incidence of NED was $34.44 \%$. The overall survival of the $\mathrm{NED}(+)$ group was significantly less favorable than that of the
\end{abstract}

Correspondence to: Professor Kefeng Ding, Department of Surgical Oncology, Key Laboratory of Cancer Prevention and Intervention, China National Ministry of Education, Key Laboratory of Molecular Biology in Medical Sciences, The Second Affiliated Hospital, Zhejiang University, School of Medicine, 88 Jiefang Road, Hangzhou, Zhejiang 310009, P.R. China

E-mail: dingkefeng@zju.edu.cn

*Contributed equally

Abbreviations: CI, confidence interval; HR, hazard ratio; CRC, colorectal cancer; NED, neuroendocrine differentiation; IHC, immunohistochemistry; Syn, synaptophysin; CgA, chromogranin A; PDCRC, poorly differentiated CRC; OS, overall survival; LND, lymph nodes discovered; MMR, DNA mismatch repair

Key words: colorectal cancer, neuroendocrine differentiation, poor survival, stage II, synaptophysin, chromogranin A
$\mathrm{NED}(-)$ group $(\mathrm{P}=0.001)$. The 5-year survival rate was $68 \%$ for $\mathrm{NED}(+)(\mathrm{n}=51)$ and $90 \%$ for $\mathrm{NED}(-)(\mathrm{n}=97)$. The independent prognostic factors of survival of patients with stage II CRC following multivariate analysis were age $\geq 65$ years $(P=0.007)$ and NED-positivity $(\mathrm{P}=0.014)$. NED was revealed to be an independent factor of poor prognosis for patients with stage II CRC, which may offer potential for improved therapy stratification.

\section{Introduction}

Colorectal cancer (CRC) is one of the most commonly diagnosed cancers worldwide with the annual incidence rising from 0.95 million in 2005 to $>1.2$ million in $2008(1,2)$. Surgical resection is the curative treatment option for the majority of local regional disease and clinical outcome is primarily dependent on tumor stage (3). According to the Surveillance, Epidemiology, and End Results (SEER) Program database, between 1992 and 2004, the 5-year survival rate for patients with stage II CRC was between 40 and $80 \%$ (3). Administration of adjuvant chemotherapy to patients with stage II CRC represents the most challenging aspect in the treatment of CRC (4). Increasing evidence has demonstrated that adjuvant therapy is associated with a disease-free survival benefit for patients with stage II CRC. However, these benefits are small and not necessarily associated with improved overall survival (4-6). The identification of independent prognostic factors may facilitate the selection of patients with stage II CRC who may benefit from adjuvant therapy.

Neuroendocrine differentiation (NED) primarily refers to the scattered or locally segregated neuroendocrine cells within the adenocarcinoma (7). The origin of neuroendocrine cells in colorectal adenocarcinoma remains unknown. Synaptophysin (Syn) and chromogranin $\mathrm{A}(\mathrm{CgA})$ are the most frequently used markers of neuroendocrine cells in CRC studies $(8,9)$. A series of retrospective studies (10-15) has illustrated that NED is a common phenomenon with an incidence rate of $\sim 40 \%$. However, its prognostic role in CRC remains controversial. In a study by Grabowski et al (14) of 146 patients with stage III and IV CRC, NED was associated with a poor prognosis. Similarly, Gulubova and Vlaykova (11) demonstrated that in 137 patients with CRC, NED was a useful marker of poor 
prognosis following surgical therapy irrespective of the tumor, node, metastasis (TNM) staging. Conversely, Foley et al (15) studied 48 patients with stage III CRC and identified no prognostic value for NED, which was consistent with the study by Cho et al (10) of 89 patients with stage II CRC.

The authors previously analyzed 171 cases of poorly differentiated CRC (PDCRC), of which 41 were stage II, and attempted to set a novel definition of NED (16); the results demonstrated that NED was not a prognostic factor. However, in the 41 cases of stage II PDCRC, NED-positivity was associated with a poor prognosis. The aim of the present study was to confirm that NED is an important prognostic factor in stage II PDCRC, and in moderately and well differentiated stage II CRC. The sample size of the previous study was limited (16). Therefore, in the present study, a total of 151 patients with stage II CRC from The Second Affiliated Hospital of Zhejiang University (Hangzhou, China) were enrolled and analyzed, and a Cox's regression test was used to assess the prognostic value of NED and supplement the TNM staging system, which may improve treatment strategies of stage II CRC.

\section{Materials and methods}

Patients and methods. All patients included received radical surgery in The Second Affiliated Hospital of Zhejiang University between January 2002 and March 2011, who were diagnosed pathologically with stage II CRC. Patient details are provided in Table I.

Potential prognostic factors were recorded: Age, gender, primary tumor location, pathological differentiation, $\mathrm{T}$ stage, lymph node number following surgery (LND) and adjuvant chemotherapy received. All these data were collected through the patients' case reviews. Survival time was measured as the time between surgery and the date of mortality, or the date of final follow-up. The end-point was set as 1 April 2013. Patients for whom there was no follow-up or died perioperatively ( \pm 3 months of the date of the surgery) were excluded. The present study was carried out according to the requirements of The Second Affiliated Hospital of Zhejiang University, School of Medicine Ethics Committee, and the patients involved in the study provided written informed consent.

Immunohistochemical (IHC) analysis of NED and DNA mismatch repair (MMR) status. Paraffin-embedded tissue sections (thickness, $4 \mu \mathrm{m}$ ) were immunostained using a two-step method. Following deparaffinization with xylene, rehydration through graded ethanol and antigen retrieval using EDTA $\left(100^{\circ} \mathrm{C}\right.$ for $\left.15 \mathrm{~min}\right)$, endogenous peroxidase activity and non-specific antigen-binding sites were blocked by successive incubation with $3 \%$ hydrogen peroxide for $10 \mathrm{~min}$ and with $5 \%$ bovine serum albumin (Bio-Rad Laboratories, Inc., Hercules, CA, USA) for $30 \mathrm{~min}$ at room temperature. The tissue sections were then incubated with each monoclonal antibody [rabbit anti-CgA monoclonal antibody (Epitomics, Burlingame, CA, USA; cat. no. AC-0037; dilution, 1:200), rabbit anti-Syn monoclonal antibody (Epitomics; cat. no. AC-0163; dilution, 1:200), mouse anti-MutL homolog 1 (MLH1) monoclonal antibody (Dako; Agilent Technologies, Inc., Santa Clara, CA, USA; clone, ES05; cat. no. M3640; dilution, 1:500), mouse anti-MutS homolog 2 (MSH2) monoclonal antibody (Abnova, Taipei,
Taiwan; clone, 25D12; cat. no. MAB9590; dilution, 1:200), rabbit anti-MSH6 monoclonal antibody (Dako; Agilent Technologies, Inc.; clone, EP49; cat. no. M3646; dilution, 1:200) and rabbit anti-post-meiotic segregation increased 1 homolog 2 (PMS2) monoclonal antibody (Dako; Agilent Technologies, Inc.; clone EP51; cat. no. M3647; dilution, 1:400)] at $37^{\circ} \mathrm{C}$ for $2 \mathrm{~h}$. Bound antibodies were detected using the REAL ${ }^{\mathrm{TM}}$ EnVision $^{\mathrm{TM}}$ Detection System (horseradish peroxidase/diaminobenzidine+, rabbit/mouse; Dako; Agilent Technologies, Inc.; cat. no. K5007), and the sections were counterstained with hematoxylin for $2 \mathrm{~min}$ at room temperature. Negative controls were prepared by following the same procedure without the primary antibody staining. Two senior pathologists evaluated the slides. The presence of CgA- and Syn-immunoreactive tumor cells was evaluated in all tumor fields. When no immunoreactive tumor cells for CgA and Syn were noted, the tumor was designated NED(-). When there were $\geq 1$ Syn- and/ or CgA-positive tumor cells/high-power field (HPF) and there was a neuroendocrine component of $<30 \%$ tumor cells/HPF, the tumor was designated $\mathrm{NED}(+)$ (Fig. 1). Furthermore, the MMR status was evaluated. If the sample was negative for anti-MLH1, anti-MSH2, anti-MSH6 or anti-PMS2 antibody, then it was described as deficient MMR (d-MMR), otherwise it was identified as proficient MMR (p-MMR).

Statistical analysis. Statistical analyses were performed using SPSS software (version 18.0; SPSS, Inc., Chicago, IL, USA). Results for the population are presented as percentage, medians and $95 \%$ confidence interval $(\mathrm{CI})$. Overall survival (OS) curves were calculated using the Kaplan-Meier estimator method. Prognostic factors were examined using a univariate and multivariate Cox's proportional hazards models. $\mathrm{P}<0.05$ was considered to indicate a statistically significant difference.

\section{Results}

Baseline characteristics. A total of 151 patients were retrospectively identified from databases of The Second Affiliated Hospital of Zhejiang University. Table I presents the baseline characteristics of patients. Median age at inclusion was 64.5 years (range, 26-89 years). Median follow-up was 56.11 months (range, 5.52-118.80 months). At the end-point, 31 patients had died. NED was present in 52/151 patients with stage II CRC (34.44\%). In addition, NED was identified by staining for Syn alone in 27/151 tumors (17.88\%), by $\mathrm{CgA}$ staining alone in $9 / 151$ tumors (5.96\%), and by Syn and $\mathrm{CgA}$ staining in 16/151 tumors (10.60\%).

Analysis of the survival stratified by patient and tumor characteristics. Survival was initially analyzed and compared with the following patient and tumor features: Age, gender, tumor location, pre-operative carcinoembryonic antigen (CEA) level, T stage and tumor differentiation, LND, MMR status and receipt of chemotherapy (Table II).

Gender distribution, tumor location, MMR status, T stage, pre-operative CEA level, tumor differentiation, and receipt of chemotherapy were not associated with statistically significant differences in prognosis. A cut-off point of 65 years of age was established. Patients $>65$ years old exhibited a significantly less favorable prognosis $(\mathrm{P}<0.001)$. Furthermore, the 
Table I. Baseline patient characteristics, and data about different clinicopathological characteristics in different synaptophysin, chromogranin A and neuroendocrine differentiation groups.

\begin{tabular}{|c|c|c|c|c|c|c|c|}
\hline \multirow[b]{2}{*}{ Characteristic } & \multirow[b]{2}{*}{$\mathrm{n}$} & \multicolumn{2}{|c|}{ Syn status } & \multicolumn{2}{|c|}{ CgA status } & \multicolumn{2}{|c|}{ NED status } \\
\hline & & + & - & + & - & + & - \\
\hline Total & 151 & 43 & 108 & 25 & 126 & 52 & 99 \\
\hline Age $\geq 65$ years & 76 & 23 & 53 & 13 & 63 & 29 & 47 \\
\hline Male & 91 & 31 & 60 & 15 & 76 & 32 & 59 \\
\hline Tumor located in colon & 106 & 27 & 79 & 12 & 94 & 31 & 75 \\
\hline $\mathrm{LND} \geq 12$ & 97 & 29 & 68 & 10 & 87 & 31 & 66 \\
\hline T4 stage & 66 & 16 & 50 & 8 & 58 & 19 & 47 \\
\hline Pre-operative serum CEA $>5 \mathrm{ng} / \mathrm{ml}$ & 44 & 12 & 32 & 6 & 38 & 15 & 29 \\
\hline d-MMR & 42 & 9 & 33 & 1 & 41 & 9 & 33 \\
\hline Poor differentiation & 32 & 7 & 25 & 2 & 30 & 9 & 23 \\
\hline Receiving chemotherapy & 57 & 11 & 46 & 13 & 44 & 17 & 40 \\
\hline
\end{tabular}

LND, lymph nodes discovered; T4 stage, fourth tumor stage; CEA, carcinoembryonic antigen; d-MMR, deficient mismatch repair.

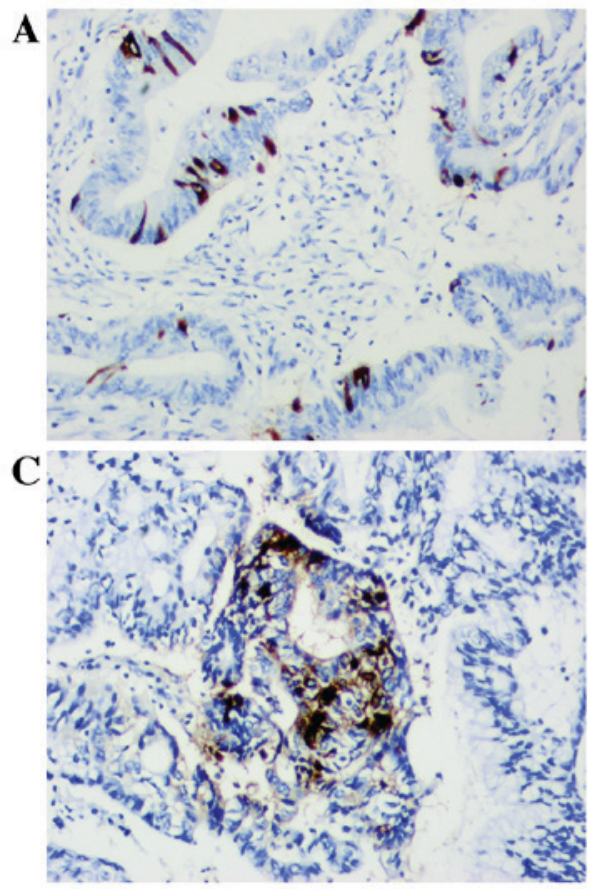

B

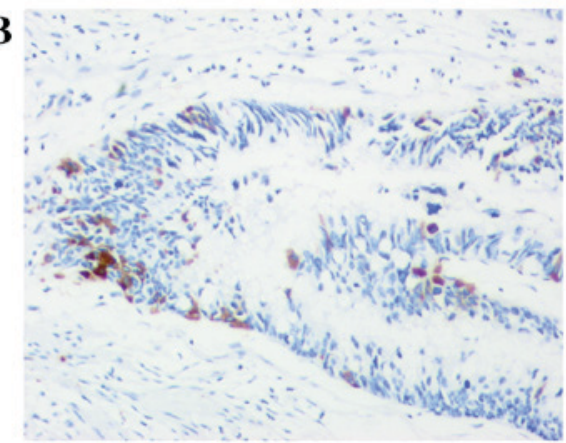

D

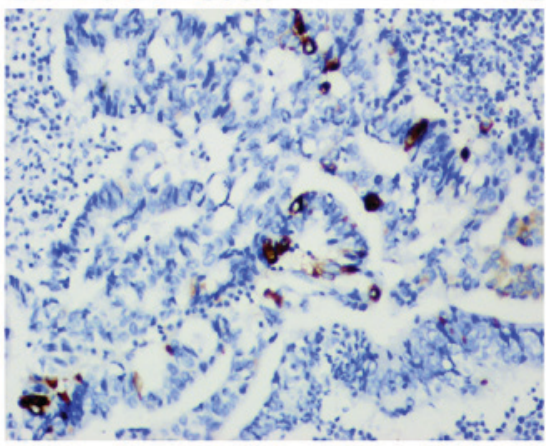

Figure 1. Immunohistochemical staining of (A and B) chromogranin A and (C and D) synaptophysin. Magnification, $\mathrm{x} 200$

OS was significantly increased in association with increased LND ( $\mathrm{P}=0.023)$ : When LND $<12$, the 5-year survival was $70 \%$, whereas when LND $\geq 12$, it was $90 \%$. Patients with T4 stage CRC and having a pre-operative CEA level $\geq 5 \mathrm{ng} / \mathrm{ml}$ appeared to have a less favorable prognosis, but no significant difference was identified $(\mathrm{P}>0.05)$.

Analysis of the survival stratified by NED markers. In the $\mathrm{NED}(+)$ group, there were 52 patients, 16 of whom died during the follow-up. The 5-year survival rate was $69 \%$, which was significantly decreased compared with the 5-year survival rate of $86 \%$ for the $\mathrm{NED}(-)$ group $(\mathrm{P}=0.001)$, which contained 99 patients, 14 of who died during the follow-up.
Furthermore, the prognostic value of the NED markers Syn and CgA was analyzed. Differences in survival between the Syn(+) group and the Syn(-) group were statistically significant $(\mathrm{P}=0.003)$. For the $\mathrm{Syn}(+)$ group, the 5-year survival was $60 \%$, whereas it was $90 \%$ for the Syn(-) group. Similar results were revealed for the $\mathrm{CgA}(+)$ group (5-year survival, 50\%) and the $\mathrm{CgA}(-)$ group (5-year survival, 88\%) ( $\mathrm{P}=0.007$; Fig. 2).

Multivariate analysis of survival. To assess the independent prognostic information, all variables that may affect the OS were included in a Cox's proportional hazards model (Table III). Independent prognostic factors were: Age $\geq 65$ [hazard ratio (HR) 4.764; 95\% CI (1.520-14.935); $\mathrm{P}=0.007$ ] and 
Table II. Univariate analysis of prognostic factors for overall survival.

\begin{tabular}{|c|c|c|c|c|}
\hline \multirow[b]{2}{*}{ Characteristic } & \multicolumn{2}{|c|}{ Patient } & \multicolumn{2}{|c|}{ Kaplan-Meier, log-rank test } \\
\hline & $\mathrm{n}$ & $\%$ & 5 -Year survival rate, $\%$ & P-value \\
\hline Age, years & & & & $<0.001^{\mathrm{b}}$ \\
\hline$<65$ & 75 & 61.59 & 90 & \\
\hline$\geq 65$ & 76 & 38.41 & 62 & \\
\hline Gender & & & & 0.509 \\
\hline Male & 91 & 60.26 & 85 & \\
\hline Female & 60 & 39.74 & 78 & \\
\hline Tumor location & & & & 0.709 \\
\hline Right colon & 55 & 36.42 & 85 & \\
\hline Left colon & 26 & 17.22 & 80 & \\
\hline Sigmoid and rectum & 70 & 46.36 & 82 & \\
\hline Pre-operative CEA level $(\mathrm{ng} / \mathrm{ml})^{\mathrm{c}}$ & & & & 0.086 \\
\hline$<5$ & 84 & 55.63 & 90 & \\
\hline$\geq 5$ & 44 & 29.14 & 80 & \\
\hline Differentiation & & & & 0.739 \\
\hline Good & 15 & 9.93 & 90 & \\
\hline Moderate & 104 & 68.87 & 80 & \\
\hline Poor & 32 & 21.19 & 86 & \\
\hline T stage & & & & 0.110 \\
\hline $\mathrm{T} 3$ & 85 & 56.29 & 85 & \\
\hline $\mathrm{T} 4$ & 66 & 43.71 & 78 & \\
\hline LND & & & & $0.023^{\mathrm{a}}$ \\
\hline$<12$ & 47 & 31.13 & 70 & \\
\hline$\geq 12$ & 104 & 68.87 & 90 & \\
\hline Receipt of chemotherapy & & & & 0.945 \\
\hline Yes & 57 & 37.75 & 80 & \\
\hline No & 94 & 62.25 & 83 & \\
\hline MMR status & & & & 0.103 \\
\hline p-MMR & 109 & 72.19 & 80 & \\
\hline d-MMR & 42 & 27.81 & 92 & \\
\hline IHC for Syn & & & & $0.003^{\mathrm{b}}$ \\
\hline Positive & 43 & 28.48 & 60 & \\
\hline Negative & 108 & 71.52 & 90 & \\
\hline $\mathrm{IHC}$ for $\mathrm{CgA}$ & & & & $0.007^{\mathrm{b}}$ \\
\hline Positive & 25 & 16.56 & 50 & \\
\hline Negative & 126 & 83.44 & 88 & \\
\hline
\end{tabular}

${ }^{\mathrm{a}} \mathrm{P}<0.05$; ${ }^{\mathrm{b}} \mathrm{P}<0.01$, for each characteristic; ${ }^{\mathrm{C}} 23$ patients did not have data for CEA. CEA, carcinoembryonic antigen; T, tumor; LND, lymph nodes discovered; MMR, DNA mismatch repair; p-MMR, proficient MMR; d-MMR, deficient MMR; IHC, immunohistochemistry; Syn, synaptophysin; CgA, chromogranin A.

NED-positivity [HR 3.238; 95\% CI (1.266-8.283); $\mathrm{P}=0.014$ ] (Table III).

\section{Discussion}

Adjuvant therapy decisions require the physician and patient to balance an understanding of the patient's likelihood of recurrence and expected absolute treatment benefit with the patient's comorbidities, life expectancy and toxicities of therapy. This is particularly the case for stage II CRC, which has a 5-year survival rate of between 40 and $80 \%$ (3). According to the 2015 National Comprehensive Cancer Network (NCCN) (Fort Washington, PA, USA) guidelines (17) for colon cancer and rectal cancer, perforation, obstruction, lymphovascular and perineural invasion, T4 stage, poor differentiation, the presence of $<12$ lymph nodes and margin-positivity in the 
Table III. Independent prognostic factors in multivariate analysis for overall survival in patients with stage II colorectal cancer.

\begin{tabular}{lcccc}
\hline & \multicolumn{4}{c}{ Overall survival } \\
\cline { 2 - 5 } Factor & $\mathrm{n}$ & HR & $95 \%$ CI & P-value \\
\hline Age $\geq 65$, years & 151 & 4.764 & $1.520-14.935$ & $0.007^{\mathrm{a}}$ \\
NED-positivity & 151 & 3.238 & $1.266-8.283$ & $0.014^{\mathrm{a}}$ \\
\hline
\end{tabular}

a $\mathrm{P}<0.05$; HR, hazard ratio; $\mathrm{CI}$, confidence interval; NED, neuroendocrine differentiation.

resection specimen are commonly recognized high-risk factors of stage II CRC, for which the patients should receive chemotherapy. However, the benefit is no more than 5\% (17). Molecular markers serve an increasingly important role in the clinical decision. A study by Chiu (17) demonstrated that somatic alterations are common in advanced CRC, with the most common alterations occurring in GTPase KRas (KRAS), phosphatidylinositol-4,5-bisphosphate 3-kinase catalytic subunit $\alpha$, tumor protein p53, adenomatous polyposis coli, phosphatase and tensin homolog deleted on chromosome 10 and mothers against decapentaplegic homolog 4. A population-based study conducted by Phipps (18) indicated that CRC subtype classifications on the basis of integrated pathways, including microsatellite instability, the $\mathrm{CpG}$ island methylator phenotype and somatic mutations in serine/threonine protein kinase B-Raf and KRAS, were associated with marked differences in survival, highlighting the significance of molecular heterogeneity in CRC. For stage II CRC, the NCCN guidelines recommend the examination of MMR status, as d-MMR provides an improved prediction of prognosis and the patient may avoid chemotherapy. In the present study, clinical risk factors were considered. The incidence of perforation $(0 / 151)$, obstruction (1/151), lymphovascular invasion (2/151) and perineural invasion (1/151) were low in stage II CRC. Therefore, the following factors were analyzed: Age, gender, tumor location, pre-operative CEA level, tumor tissue differentiation, $\mathrm{T}$ stage, LND, receipt of chemotherapy, MMR status, and the IHC of Syn and $\mathrm{CgA}$. Despite an apparent improved survival of patients with T3 stage CRC exhibiting d-MMR, no significant survival difference between d-MMR and p-MMR $(\mathrm{P}=0.103)$, or between T3 and T4 stage $(\mathrm{P}=0.110)$, was identified. This differed from other studies $(19,20)$, which demonstrated that d-MMR and T3 stage have survival benefits. This may be partially due to the sample size and discrimination of $\mathrm{T}$ stage by different pathologists.

In the present study, two independent factors of stage II CRC were included in the multivariate analysis: Age $\geq 65$ years and NED-positivity. For the age, the result of the present study is consistent with that of Steele et al (21), who collected 101,767 stage I, II and III colon adenocarcinoma samples from the SEER database and identified that the mean lymph node yield decreased with increasing age $(\mathrm{P}<0.001)$, and identified that the overall survival of older patients is less favorable than that of younger patients with stage II colon cancer. NED represents heterogeneity of tumor
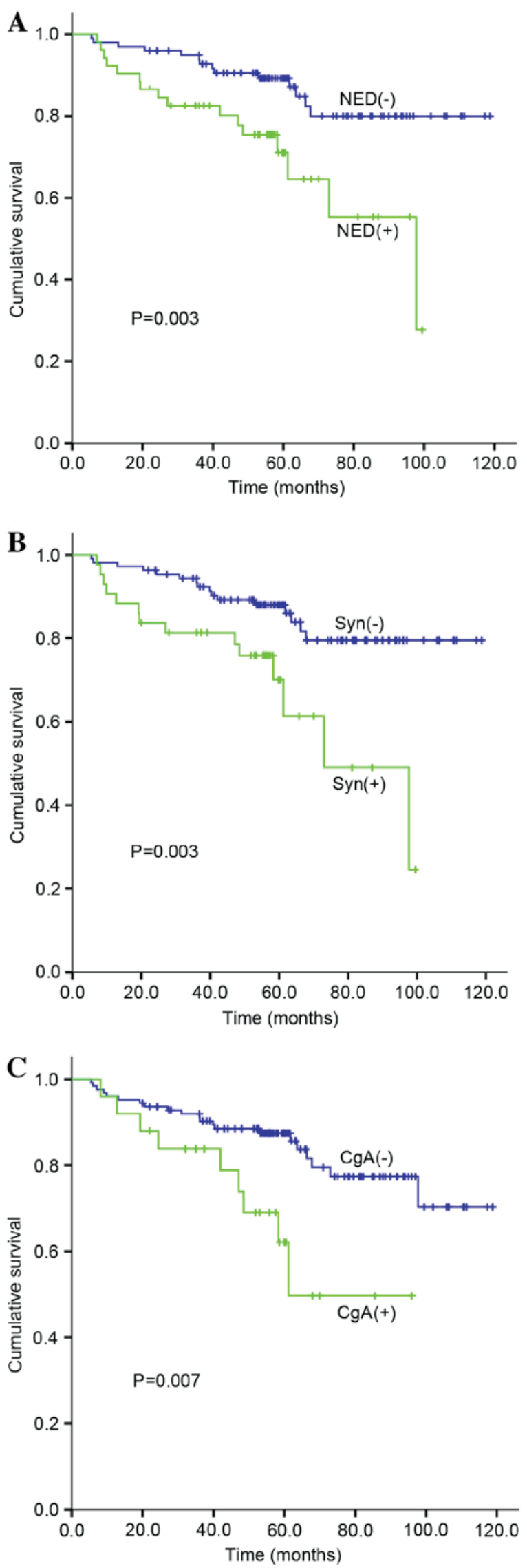

Figure 2. Overall survival of patients with stage II colorectal cancer according to NED status. (A) The cumulative survival difference between $\mathrm{NED}(+)$ and $\mathrm{NED}(-)$ groups $(\mathrm{P}=0.003)$. (B) The cumulative survival difference between $\operatorname{Syn}(+)$ and $\operatorname{Syn}(-)$ groups $(\mathrm{P}=0.003)$. (C) The cumulative survival difference between $\mathrm{CgA}(+)$ and $\mathrm{CgA}(-)$ groups $(\mathrm{P}=0.007)$. NED, neuroendocrine differentiation; Syn, synaptophysin; CgA, chromogranin A. 
cells with unclear origin and results. the authors previously defined NED-positivity as having a neuroendocrine cell component of $<30 \% / \mathrm{HPF}$ and $\geq 1$ Syn- or CgA-positive tumor cells/HPF (16). In the present study, using univariate and multivariate analysis, NED-positivity was identified as an independent prognostic factor of stage II CRC. It is consistent with the study by Gulubova and Vlaykova (11), which demonstrated that NED was a poor prognostic factor in 137 cases of stage I-IV CRC, particularly in early-stage cancer (stage I and stage II). By contrast, other studies $(10,12-15)$ have revealed different results, which may be associated with changes in NED definition. For instance, for mixed adenoneuroendocrine carcinoma, a neuroendocrine cell component of $>30 \%$ / HPF may not be defined as NED-positive. Therefore, further studies are required for the novel standard of NED, and its prognostic implication should be further investigated and verified. In the present study, it is proposed that NED is an independent factor of poor prognosis, and that $\mathrm{CgA}$ and Syn are important molecular markers of poor prognosis in stage II CRC.

Furthermore, the underlying molecular mechanism for NED as a factor of poor prognosis in stage II CRC remains unclear. Neuroendocrine tumor cells are characterized by cytoplasmic dense-core neurosecretory granules synthesizing neuropeptides and receptors (22). These autocrine/ paracrine mechanisms involving the release of neuropeptides may contribute to drug responsiveness and disease progression $(16,23)$. In the small cell lung cancer cell line A549, NED occurred during the development of resistance to an epidermal growth factor receptor tyrosine kinase inhibitor (24), and Syn and CgA expression made the cells more sensitive to chemotherapy (25). However, in esophageal and esophagogastric junction adenocarcinomas, tumor cells with NED were more resistant to neoadjuvant chemoradiation (26). These results indicate that NED is associated with drug resistance and sensitivity. By contrast, in the present study, in stage II CRC, the NED-positive rate was $34.44 \%$. In stage III and stage IV CRC, the NED-positive rate was $54.55 \%(90 / 165)$ and $64.29 \%$ (63/98), respectively. The CgA- and Syn-positive rates were $16.56 \%(25 / 151)$ and $28.48 \%(43 / 151)$ in stage II, $24.86 \%(41 / 165)$ and $49.70 \%(82 / 165)$ in stage III, and $36.73 \%$ (36/98) and $60.20 \%$ (59/98) in stage IV, respectively. These results indicate that NED is associated with tumor progression in CRC. However, with the increasing rate of pre-operation chemotherapy in stage III and stage IV, it remains unclear whether chemotherapy resistance or tumor cell progression causes the high incidence of NED. In the present study, no association was identified between NED and receipt of chemotherapy in stage II CRC, possibly due to the small number of patients receiving chemotherapy. Additional studies on CRC are required before a full understanding of any association between NED in CRC cells and response to therapy can be determined.

In conclusion, NED is an independent prognostic factor of poor survival outcome in patients with stage II CRC. In particular, expression of Syn is associated with poor survival, which may offer the potential for improved therapy stratification. Further research is necessary to elucidate the underlying molecular mechanisms of this phenomenon and to develop targeted therapies against NED in CRC.

\section{Acknowledgements}

The authors of the present study thank Haijun Wang and Lifang Yao in the Department of Pathology, The Second Affiliated Hospital, Zhejiang University, School of Medicine (Hangzhou, China), for technical assistance. The present study was supported by the Research Fund of Public Welfare in the Health Industry, 2014, Health Ministry of China (grant no. 201402015) and Zhejiang Provincial Natural Science Foundation of China (grant no. LY13H160017).

\section{References}

1. Jemal A, Bray F, Center MM, Ferlay J, Ward E and Forman D: Global cancer statistics. CA Cancer J Clin 61: 69-90, 2011.

2. Weitz J, Koch M, Debus J, Höhler T, Galle PR and Büchler MW: Colorectal cancer. Lancet 365: 153-165, 2005.

3. Gunderson LL, Jessup JM, Sargent DJ, Greene FL and Stewart AK: Revised TN categorization for colon cancer based on national survival outcomes data. J Clin Oncol 28: 264-271, 2010.

4. Gill S, Loprinzi CL, Sargent DJ, Thomé SD, Alberts SR, Haller DG, Benedetti J, Francini G, Shepherd LE, Francois Seitz J, et al: Pooled analysis of fluorouracil-based adjuvant therapy for stage II and III colon cancer: Who benefits and by how much? J Clin Oncol 22: 1797-1806, 2004.

5. Figueredo A, Charette ML, Maroun J, Brouwers MC and Zuraw L: Adjuvant therapy for stage II colon cancer: A systematic review from the cancer care ontario program in evidence-based care's gastrointestinal cancer disease site group. J Clin Oncol 22: 3395-3407, 2004.

6. Benson AB III, Schrag D, Somerfield MR, Cohen AM, Figueredo AT, Flynn PJ, Krzyzanowska MK, Maroun J, McAllister P, Van Cutsem E, et al: American society of clinical oncology recommendations on adjuvant chemotherapy for stage II colon cancer. J Clin Oncol 22: 3408-3419, 2004.

7. Kleist B and Poetsch M: Neuroendocrine differentiation: The mysterious fellow of colorectal cancer. World J Gastroenterol 21: $11740-11747,2015$

8. Wiedenmann B, Franke WW, Kuhn C, Moll R and Gould VE: Synaptophysin: A marker protein for neuroendocrine cells and neoplasms. Proc Natl Acad Sci USA 83: 3500-3504, 1986.

9. Hamada Y, Oishi A, Shoji T, Takada H, Yamamura M, Hioki K and Yamamoto M: Endocrine cells and prognosis in patients with colorectal carcinoma. Cancer 69: 2641-2646, 1992.

10. Cho YB, Yang SS, Lee WY, Song SY, Kim SH, Shin HJ, Yun SH and Chun HK: The clinical significance of neuroendocrine differentiation in T3-T4 node-negative colorectal cancer. Int J Surg Pathol 18: 201-206, 2010.

11. Gulubova M and Vlaykova T: Chromogranin A-, serotonin-, synaptophysin- and vascular endothelial growth factor-positive endocrine cells and the prognosis of colorectal cancer: An immunohistochemical and ultrastructural study. J Gastroenterol Hepatol 23: 1574-1585, 2008.

12. Shinji S, Naito Z, Ishiwata T, Tanaka N, Furukawa K, Suzuki H, Seya T, Kan H, Tsuruta H, Matsumoto S, et al: Neuroendocrine cell differentiation of poorly differentiated colorectal adenocarcinoma correlates with liver metastasis. Int J Oncol 29: 357-364, 2006.

13. Grabowski P, Sturm I, Schelwies K, Maaser K, Buhr HJ, Dörken B, Zeitz M, Daniel PT and Scherübl H: Analysis of neuroendocrine differentiation and the p53/BAX pathway in UICC stage III colorectal carcinoma identifies patients with good prognosis. Int J Colorectal Dis 21: 221-230, 2006.

14. Grabowski P, Schindler I, Anagnostopoulos I, Foss HD, Riecken EO, Mansmann U, Stein H, Berger G, Buhr HJ and Scherübl H: Neuroendocrine differentiation is a relevant prognostic factor in stage III-IV colorectal cancer. Eur J Gastroenterol Hepatol 13: 405-411, 2001.

15. Foley EF, Gaffey MJ and Frierson HF Jr: The frequency and clinical significance of neuroendocrine cells within stage III adenocarcinomas of the colon. Arch Pathol Lab Med 122: 912-914, 1998.

16. Liu Y, Xu J, Jiao Y, Hu Y, Yi C, Li Q, Tong Z, Wang X, Hu L, $\mathrm{Xiao} \mathrm{Q}$, et al: Neuroendocrine differentiation is a prognostic factor for stage II poorly differentiated colorectal cancer. Biomed Res Int 2014: 789575, 2014. 
17. Provenzale D, Jasperson K, Ahnen DJ, Aslanian H, Bray T, Cannon JA, David DS, Early DS, Erwin D, Ford JM, et al: Colorectal cancer screening, version 1.2015. J Natl Compr Canc Netw 13: 959-968, 2015.

18. Chiu JW: Molecular profiling of patients (pts) with advanced colorectal cancer (CRC): Princess Margaret Cancer Centre experience. Gastrointestinal Cancers Symposium 32 (Suppl 3) abstr 459, 2014.

19. Phipps AI: Molecular subtypes of colorectal cancer in relation to disease survival. Gastrointestinal Cancers Symposium 32 (Suppl 3): abstr 451, 2014.

20. Sargent DJ, Marsoni S, Monges G, Thibodeau SN, Labianca R Hamilton SR, French AJ, Kabat B, Foster NR, Torri V, et al: Defective mismatch repair as a predictive marker for lack of efficacy of fluorouracil-based adjuvant therapy in colon cancer. J Clin Oncol 28: 3219-3226, 2010.

21. Steele SR, Chen SL, Stojadinovic A, Nissan A, Zhu K, Peoples GE and Bilchik A: The impact of age on quality measure adherence in colon cancer. J Am Coll Surg 213: 95-105, 2011.
22. Chaudhry A, Funa $\mathrm{K}$ and Oberg K: Expression of growth factor peptides and their receptors in neuroendocrine tumors of the digestive system. Acta Oncol 32: 107-114, 1993.

23. Shenoy S: Gastrointestinal carcinoids and colorectal cancers: Is it a paracrine effect? Tumori 99: e141-e143, 2013

24. Bertagnolli MM, Redston M, Compton CC, Niedzwiecki D, Mayer RJ, Goldberg RM, Colacchio TA, Saltz LB and Warren RS: Microsatellite instability and loss of heterozygosity at chromosomal location 18q: Prospective evaluation of biomarkers for stages II and III colon cancer-a study of CALGB 9581 and 89803. J Clin Oncol 29: 3153-3162, 2011.

25. Gangadhar T and Schilsky RL: Molecular markers to individualize adjuvant therapy for colon cancer. Nat Rev Clin Oncol 7: 318-325, 2010.

26. Wang KL, Yang Q, Cleary KR, Swisher SG, Correa AM, Komaki R, Ajani JA, Rashid A, Hamilton SR and Wu TT: The significance of neuroendocrine differentiation in adenocarcinoma of the esophagus and esophagogastric junction after preoperative chemoradiation. Cancer 107: 1467-1474, 2006. 